\title{
Characterization of Carbohydrate Fermentation in Feces of Formula-Fed and Breast-Fed Infants ${ }^{1}$
}

\author{
CARLOS H. LIFSCHITZ, MEYER J. WOLIN, ${ }^{2}$ AND PETER J. REEDS \\ USDA/ARS Children's Nutrition Research Center, Department of Pediatrics, Baylor College of Medicine and \\ Texas Children's Hospital, Houston, Texas 77030
}

\begin{abstract}
We wished to characterize the carbohydrate fermentation by intestinal flora in formula-fed infants and in breast-fed infants. We also wished to compare the carbohydrate fermentation process in the two groups to determine whether differences that existed between groups could help explain the observation that breast-fed infants usually have milder forms of acute gastroenteritis. We performed in vitro incubations of fecal samples from nine formula-fed and 14 breast-fed infants and examined the samples for fermentation characteristics, the effect of acid pH on bacterial fermentation, and changes in carbohydrate fermentation in relation to the age of the infant. Fecal samples were incubated, with and without the addition of lactose, at a pH of 6.8 and at a $\mathrm{pH}$ of 5.5. Volatile fatty acids and carbohydrates were determined in the incubates. The addition of lactose to the incubate at $\mathrm{pH} 6.8$ resulted in significantly increased volatile fatty acid production and larger amounts of lactose, glucose, and galactose compared with the values observed in 1-h incubates to which no lactose was added. At pH 5.5, volatile fatty acid production was significantly lower in both groups compared with that at pH 6.8, and the accumulation of monosaccharides in the incubate of feces of formula-fed infants increased significantly $(p<0.05)$. In contrast, in stools from breast-fed infants, incubation at $\mathbf{p H} 5.5$ resulted in a greater proportion of saccharides as lactose, i.e. a decrease in the amount of lactose hydrolyzed $(p<0.01$ ), accompanied by a nonsignificant increase in the amount of hexose. The decrease in lactose hydrolysis in breast-fed infants results in a lower luminal osmolar load, which may provide a partial explanation for the fact that acute gastroenteritis is milder in this population. (Pediatr Res 27: 165-169, 1990)
\end{abstract}

\section{Abbreviations}

VFA, volatile fatty acids

CHO, carbohydrate

Although there is substantial variation among infants in the type and number of bacteria in the feces, a number of studies have demonstrated qualitative differences in the fecal flora of

Received July 6, 1989; accepted September 29, 1989.

Correspondence and reprint requests Carlos H. Lifschitz, M.D., Children's Nutrition Research Center, 1100 Bates Street, Houston, TX 77030.

Supported in part with federal funds from the U.S. Department of Agriculture, Agricultural Research Service under Cooperative Agreement 58-7MN1-6-100.

'Publication of the USDA/ARS Children's Nutrition Research Center, Department of Pediatrics, Baylor College of Medicine and Texas Children's Hospital, Houston, TX. The contents of this publication do not necessarily reflect the views or policies of the U.S. Department of Agriculture, nor does mention of trade names, commercial products, or organizations imply endorsement by the U.S. Government.

${ }^{2}$ Laboratory of Environmental Sciences, Wadsworth Center for Laboratories and Research, State Department of New York, Albany, NY 12201. infants who have been fed human milk compared with that of infants fed formulas. Some studies have found a predominance of bifidobacteria in the feces of breast-fed infants (1-3), but others have questioned this observation, especially in breast-fed infants born in modern hospitals $(4,5)$.

Different bacteria ferment $\mathrm{CHO}$ by different pathways, which provides an opportunity for characterization of the fecal flora on the basis of its metabolic properties $(6,7)$. This approach to the characterization of the flora also provides information on bacterial function, i.e. the formation of VFA, which has immediate physiologic and function importance for the host.

Our objectives were to 1 ) investigate whether differences in the fermentation patterns of bacteria could provide part of the explanation for the fact that diarrhea in breast-fed infants is less severe than in formula-fed infants, 2) examine the effect that acid $\mathrm{pH}$ has on bacterial fermentation of carbohydrate, and 3 ) determine whether age-related differences exist in the scale and quantity of lactose fermentation and whether, in turn, these differences are related to the type of feeding of the infant. We therefore performed in vitro carbohydrate fermentation studies with the feces of formula-fed and breast-fed infants, with and without the addition of lactose, at $\mathrm{pH} 6.8$, as in stools of normal

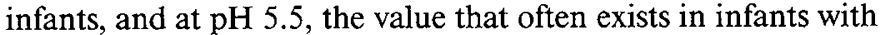
diarrhea and carbohydrate malabsorption. We measured the accumulation of hexoses and VFA.

\section{MATERIALS AND METHODS}

Feces were collected only from infants who were exclusively either formula-fed or breast-fed. All infants had been delivered vaginally, and none had any illness or gastrointestinal problems. Only two of the formula-fed infants had received antibiotics and then only during their first $48 \mathrm{~h}$ after birth; at the time of the study both infants were more than 1 mo old. Neither the breastfed infants nor their mothers had received antibiotics. All infants were thriving appropriately. Eligible formula-fed infants were receiving ad libitum amounts of casein-based, proprietary formulas that contained lactose, a mixed vegetable-fat blend, and no supplemental iron (Enfamil, Bristol Myers, Evansville, IN; or Similac, Ross Labs., Columbus, OH). No infant received any supplemental feeding. The protocol was approved by the Baylor Institutional Review Board for Human Research.

Fecal collection and preparation. Stools were collected at the Clinical Research Center at Texas Children's Hospital or at the infants' homes: they were scraped from the diaper and placed in a plastic bag, kept at ambient temperature, and delivered to the laboratory. Stool processing was begun within $1 \mathrm{~h}$ of defecation. Stools were homogenized for $2 \mathrm{~min}$ in $2 \mathrm{vol}$ of $0.1 \mathrm{M}$ PBS solution (pH 7.0) with a Stomacher Lab Blender 80 (Tekmar Co., Cincinnati, $\mathrm{OH}$ ). Aliquots were divided into thirds, placed in 50-mL Vacutainers, and prepared for analysis. In the first aliquot, no carbohydrate was added, and the $\mathrm{pH}$ was adjusted to 6.8 by the addition of appropriate amounts of concentrated $\mathrm{NaOH}$; in the second aliquot, a lactose solution $(105 \mu \mathrm{mol} / \mathrm{g}$ 
stool homogenate) was added, and the $\mathrm{pH}$ was adjusted to 6.8 ; and in the third aliquot, a lactose solution $(105 \mu \mathrm{mol} / \mathrm{g}$ stool homogenate) was added, and the $\mathrm{pH}$ was adjusted to 5.5 by the addition of concentrated $\mathrm{HCl}$ or acetic acid. Fecal $\mathrm{pH}$ was determined using an Accumet pH meter, model 805 MP (Fisher Scientific, Orangeburg, NY). We were unable to collect sufficient fecal material to perform incubations without lactose at pH 5.5.

Tubes were gassed with $\mathrm{N}_{2}$ to create anaerobic conditions, capped, and incubated for $1 \mathrm{~h}$ at $37^{\circ} \mathrm{C}$. At the end of the incubation, the tubes were immediately frozen to interrupt further fermentation. These tubes were stored frozen for later analysis of VFA and CHO. In addition, a baseline stool sample, which did not undergo any incubation, was also analyzed for VFA and $\mathrm{CHO}$.

Analyses of volatile fatty acids and carbohydrate by HPLC. A 5 -mL aliquot of fecal homogenate was centrifuged at $9000 \times g$ for $20 \mathrm{~min}$ at $2^{\circ} \mathrm{C}$. The supernatant was then filtered through a microconcentrator with a $\mathrm{M}_{\mathrm{r}}$ cutoff of $3000 \mathrm{D}$, by centrifugation at $7000 \times g$ for $1 \mathrm{~h}$ at $2^{\circ} \mathrm{C}$. Three $10-\mu \mathrm{L}$ aliquots of this filtrate were then analyzed for short-chain fatty acids by ion-exclusion HPLC (Water Associates, Milford, MA). Chromatography was effected on an ORH-801 cation exchange column using 0.2 $\mathrm{mmol} / \mathrm{L}$ solution of ultrapure sulfuric acid (nominal $\mathrm{pH} 3.4$ ). The method for VFA analysis is detailed elsewhere (8). VFA were detected with a conductivity detector against standards of formic, lactic, acetic, propionic, and butyric acids.

Lactose, glucose, and galactose were separated by HPLC on a Sugar Pack 1 column in the Ca form. Elution was with water containing $0.1 \mathrm{mmol} / \mathrm{L}$ solution of Ca-EDTA. Peaks were identified with a refractive index detector. The dry weight of the stool samples was determined by drying approximately $0.5 \mathrm{~g}$ of sample in an oven $\left(95^{\circ} \mathrm{C}\right)$ until constant wt was reached.

Data analysis. To analyze the fermentation patterns in the presence of additional lactose, and in particular the distribution among bifidobacteria-type, homolactate, and propionate fermentation reactions, the data are expressed in two forms: overall carbon balance, i.e. g lactose carbon hydrolyzed and VFA carbon produced, and the pattern of fermentation, i.e. $\mu \mathrm{mol}$ of totally formed product or $\mu \mathrm{mol}$ of product per $\mu \mathrm{mol}$ fermented lactose. In both calculations, the results were adjusted for the amount of VFA produced in control incubations at $\mathrm{pH} 6.8$ with no added lactose. The total carbon in all products (lactate, formate, acetate, propionate, butyrate, and carbon dioxide) was divided by 12 , to give the molar amount of lactose required for the product array. All values were then converted to moles product produced per $100 \mathrm{~mol}$ of substrate hexose (two hexoses per lactose) fermented. This allowed a true comparison of all fermentations, regardless of fermentation equations and pathways.

The rate of fermentation was calculated directly from the quantities of VFA produced. The rate of lactose hydrolysis was calculated from the amount of lactose that remained after incubation. Measured VFA production accounted for 88 to $95 \%$ of the nonsaccharide carbon. Of the residual carbon, more than $50 \%$ could be accounted for by $\mathrm{CO}_{2}$ production (not measured), which would have been associated with the measured synthesis of propionate (reaction 2, below). To calculate the contributions of different pathways to total VFA production, we assumed that the following pathways dominated the process of fermentation (9):

$$
\begin{aligned}
& 2 \mathrm{C}_{6} \mathrm{H}_{12} \mathrm{O}_{6} \rightarrow 2 \mathrm{CH}_{3} \mathrm{CHOHCO} 2 \mathrm{H}+3 \mathrm{CH}_{3} \mathrm{CO}_{2} \mathrm{H} \\
& 2 \text { hexose } 2 \text { lactate }+3 \text { acetate (reaction } 1 \text { ) } \\
& \mathrm{C}_{6} \mathrm{H}_{12} \mathrm{O}_{6} \rightarrow \mathrm{CH}_{3} \mathrm{CH}_{2} \mathrm{CO}_{2} \mathrm{H}+\mathrm{CH}_{3} \mathrm{CO}_{2} \mathrm{H}+\mathrm{CO}_{2}+\mathrm{H}_{2} \\
& \text { hexose propionate acetate (reaction 2) }
\end{aligned}
$$

$$
\underset{\text { hexose }}{\mathrm{C}_{6} \mathrm{H}_{12} \mathrm{O}_{6}} \rightarrow \underset{2}{2 \mathrm{CH}_{3} \mathrm{CHOHCO}_{2} \mathrm{H}}
$$

The relative contributions of each pathway were calculated as follows: reaction 2 produces equimolar amounts of propionate and acetate, so that the acetate production via reaction 1 (bifidobacterial fermentation) can be calculated from the propionate production (i.e. total moles acetate $-\mathrm{mol}$ propionate $=\mathrm{mol}$ acetate produced in reaction 1). Lactate production from reaction 1 then equals $0.67 \times$ (total mol acetate - mol propionate), and the remaining lactate is derived from reaction 3 , the homolactate fermentation, i.e. total lactate $-[0.67 \times($ total acetate - propionate)].

Paired $t$ tests were used to determine the significance of differences between the various treatments of feces from infants within the same feeding group. The Mann-Whitney test was used to determine the significance of differences between incubates of feces that underwent the same treatment but were from infants of different feeding groups. The amount of lactose added to the incubates was calculated from the wet wt of the stools, therefore regression analysis was performed to determine whether the amount of added lactose influenced results based on the dry wt of the stools.

\section{RESULTS}

Feces from nine formula-fed and 14 breast-fed infants were studied. The mean ages of the two groups were similar (mean \pm SEM $67.1 \pm 18.6$ and $77.6 \pm 15.5 \mathrm{~d}$ for formula- and breast-fed, respectively, range 10 to $174 \mathrm{~d}$ ). The concentration of butyrate was so low that results of this VFA are not included in this report.

Baseline composition and incubation without lactose. The stools of the formula-fed infants contained a significantly higher proportion of dry matter than those of the breast-fed group (mean \pm SEM; $23.2 \% \pm 1.5$ versus $16.9 \% \pm 1, p<0.05$ ). The results, however, are expressed per $U$ wet wt of stool, because analyses based on per U dry wt did not alter our conclusions.

The fresh feces from breast-fed infants contained significantly more lactose than those from formula-fed infants, but there were no significant differences in the contents of hexoses or lactic acid (Table 1). Total VFA was higher $(p<0.05)$ in feces from the formula-fed group than in those from breast-fed infants (mean \pm SEM; $204 \pm 19$ versus $110 \pm 17 \mu \mathrm{mol} / \mathrm{g}$ wet feces), largely a result of the higher contents of formic (data not shown), acetic, and propionic acids in the feces of the formula-fed infants $(p<$ 0.05 ) (Table 1).

After a 1-h incubation without added substrate, the amounts of lactose and monosaccharides decreased slightly and those of lactate and VFA increased. The pattern of the VFA was unaltered, and the differences observed in the baseline samples were essentially preserved (Table 1).

One-h incubation with lactose at $p H$ 6.8. The data from incubations with added lactose (Table 1) were adjusted for values obtained from incubations without lactose (Table 1) to obtain net CHO utilization and VFA production values (Tables 2 and 3 ). Because the amount of lactose solution added to the incubates was calculated on the basis of wet stool wt, we had to determine whether the amount of lactose added may have influenced the results. Regression analysis indicated that neither the amount of lactose added nor the dry wt of the stools had an effect on the amount of $\mathrm{CHO}$ in the incubate at the end of $1 \mathrm{~h}$ at either $\mathrm{pH}$ 6.8 or 5.5 . The incubation did not result in net production of formate and the results are not included.

During the incubation, more than $90 \%$ of the added lactose was hydrolyzed (Table 2). The increase in VFA production accounted for 54 and $50 \%$ (in formula-fed and breast-fed groups, respectively) of the hydrolyzed lactose. The sum of carbon present as hexose and VFA was less than the amount of lactosecarbon hydrolyzed. The difference was $16 \%$ in formula-fed and $11 \%$ in breast-fed groups. A portion of the difference could be explained by the generation of $\mathrm{CO}_{2}$ during the fermentation process. At $\mathrm{pH} 6.8$, the fermentation capacity of the fecal flora 
was less than the capacity to hydrolyze lactose. As a result, both glucose and galactose increased to similar levels in both groups.

The mean values for lactic or acetic acid in the feces did not differ between feeding groups. The amount of propionate, however, remained significantly higher in the fecal samples of the formula-fed infants compared with those of the breast-fed infants.

One-h incubation with lactose at pH 5.5. Results from acidification of the fecal incubate with $\mathrm{HCl}$ were not significantly different from those obtained from acidification with acetic acid. Results from the incubates acidified with $\mathrm{HCl}$ are presented.

The incubation of feces from formula-fed infants at $\mathrm{pH} 5.5$ resulted in a nonsignificant decrease in the quantity of hydrolyzed lactose. Incubates of feces from the breast-fed group, however, had significantly decreased lactose hydrolysis, indicated by the significant increase in the amount of lactose that remained in the incubate. The increase in the amounts of glucose and galactose that remained in the fecal incubates of breast-fed infants $(12 \%)$ was not significant (Table 2). The increase in the amounts

Table 1. Saccharide and VFA levels in fresh and prepared* feces of formula-fed (FF) and breast-fed (BF) infants (mean \pm SEM)

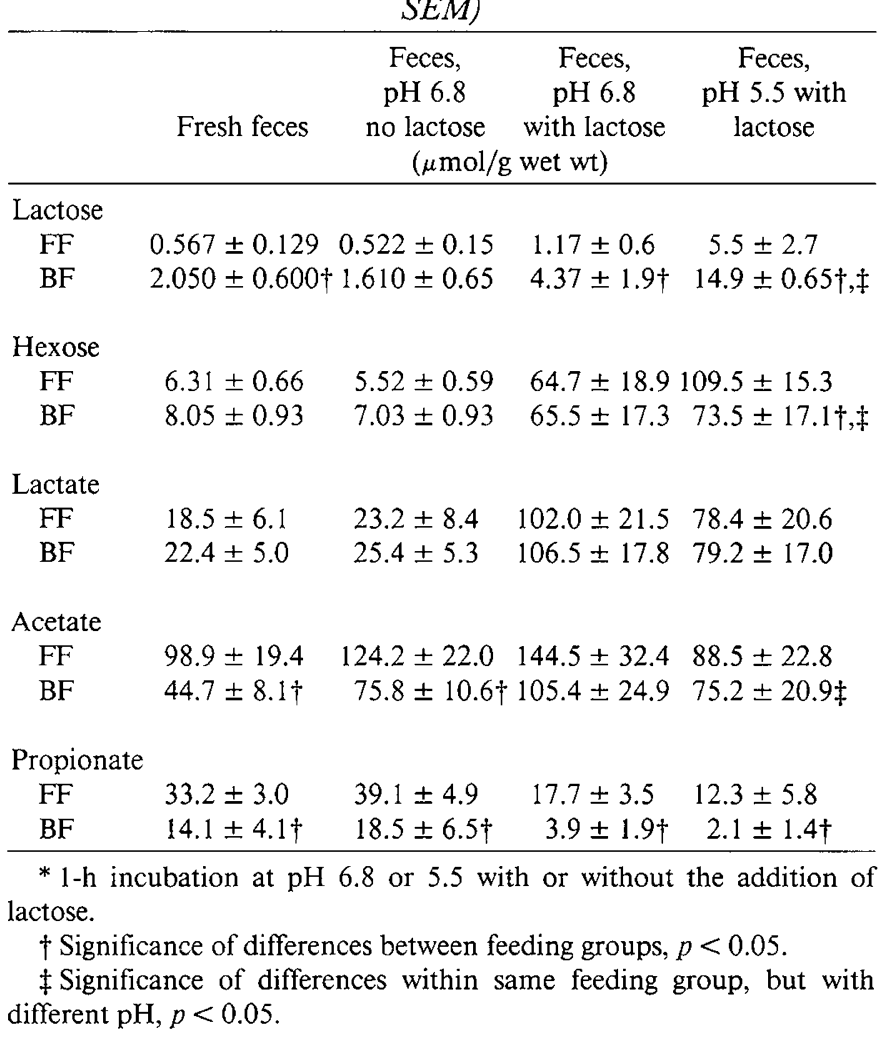

of glucose and galactose that remained in the incubates of feces of formula-fed infants $(68 \%)$ after the 1-h incubation at $\mathrm{pH} 5.5$ was significant compared with that after the 1-h incubation at $\mathrm{pH} 6.8(p<0.05)$. The amount of lactose hydrolyzed was significantly lower for breast-fed infants compared with that for formula-fed infants (Table 2).

Analyses of VFA patterns. The VFA patterns were assigned to one of the major fermentation pathways based on the three reactions described in the "Materials and Methods." This analysis suggested that, at $\mathrm{pH} 6.8,62$ to $64 \%$ of the fermentation followed the pattern of fermentation by bifidobacteria. The remainder could be accounted for by a combination of homolactate and propionate fermentation (Table 3 ). The contributions to the total fermentation from these three pathways were different between the two feeding groups. Propionic acid fermentation accounted for $25 \%$ of the fermentation in the fecal incubates of the formulafed infants, and the homolactate fermentation accounted for $29 \%$ in the incubates of the breast-fed infants. At pH 5.5, the contribution to the total fermentation by the bifidobacteria pathway decreased and that by the homolactate pathway increased. Fermentation via the homolactate pathway therefore accounted for 30 and $42 \%$ (in formula-fed and breast-fed infants, respectively) of the total. The diet-related difference in propionate fermentation remained unaffected.

Age-related changes. We found no relationship between the ages of the formula-fed infants and 1 ) the sum of VFA present in the baseline stool samples or in stool samples after a $1-\mathrm{h}$ incubation (Fig. 1), or 2) the amount of hexose present in stool samples after a 1-h incubation (Fig. 2). A significant positive correlation was found, however, between the ages of the breastfed infants and the amount of VFA present in the incubate after a 1-h incubation (regression equation: age $=26.9+0.232$ VFA, $r^{2}=33.7 \%, p=0.03$ ) (Fig. 1). We found a significant negative correlation between the ages of the breast-fed infants and the hexose accumulated after a $1-\mathrm{h}$ incubation at $\mathrm{pH} 6.8$ (regression equation: age $=111-0.92$ remaining hexose, $r^{2}=20.7 \%, p=$ 0.03) (Fig. 2).

\section{DISCUSSION}

We wished to investigate whether differences between $\mathrm{CHO}$ fermentation by the fecal flora of formula-fed infants and that of breast-fed infants could 1) help characterize the flora and 2) provide further explanation for the observation that diarrhea is generally less severe in breast-fed infants. Duffy et al. (15) demonstrated that, although breast-fed infants did not have a lower incidence of infection by rotavirus than formula-fed infants, the severity of their diarrheal illness was lower. The hygienic practices inherent in breast-feeding and the immunologic factors in human milk may be, in part, responsible for the diminished severity of diarrhea in breast-fed infants. Another factor responsible for this phenomenon may be the manner in which the fecal flora of

Table 2. Hydrolysis and fermentation of lactose ( $m g$ carbon $/ g$ wet wt/h) during incubations of feces of formula-fed (FF) and breastfed $(B F)$ infants at $p H 6.8$ and 5.5 (mean \pm SEM)

\begin{tabular}{|c|c|c|c|c|c|c|c|}
\hline & \multirow{2}{*}{$\begin{array}{c}\text { Lactose } \\
\text { hydrolyzed }\end{array}$} & \multicolumn{2}{|c|}{ Hexose } & \multirow{2}{*}{$\begin{array}{l}\text { VFA } \\
\text { Total }\end{array}$} & \multirow{2}{*}{$\begin{array}{c}\begin{array}{c}\text { Unaccounted } \\
\text { carbon }\end{array} \\
\text { Proportion }\end{array}$} & \multirow{2}{*}{$\begin{array}{l}\text { Proportion of } \\
\text { added lactose } \\
\text { hydrolyzed }\end{array}$} & \multirow{2}{*}{$\begin{array}{c}\text { Proportion o } \\
\text { hydrolyzed } \\
\text { lactose } \\
\text { fermented }\end{array}$} \\
\hline & & Remaining & Produced & & & & \\
\hline \multicolumn{8}{|c|}{ pH 6.8} \\
\hline $\mathrm{FF}$ & $14.8 \pm 0.7$ & $4.4 \pm 1.5$ & $8.0 \pm 1.4$ & $2.4 \pm 0.8$ & $0.16 \pm 0.04$ & $0.98 \pm 0.04$ & $0.54 \pm 0.09$ \\
\hline $\mathrm{BF}$ & $14.3 \pm 0.4$ & $5.5 \pm 1.2$ & $7.2 \pm 1.2$ & $1.6 \pm 0.4$ & $0.11 \pm 0.03$ & $0.94 \pm 0.02$ & $0.50 \pm 0.07$ \\
\hline \multicolumn{8}{|c|}{ pH 5.5} \\
\hline $\mathrm{FF}$ & $14.6 \pm 0.4^{*}$ & $8.1 \pm 1.2 \dagger$ & $5.5 \pm 1.1 \dagger$ & $1.0 \pm 0.7$ & $0.06 \pm 0.03$ & $0.97 \pm 0.03^{*}$ & $0.37 \pm 0.09 \dagger$ \\
\hline $\mathrm{BF}$ & $12.2 \pm 0.4 \dagger$ & $6.4 \pm 1.3$ & $4.8 \pm 1.1 \dagger$ & $1.0 \pm 0.3$ & $0.08 \pm 0.03$ & $0.80 \pm 0.03 \dagger$ & $0.39 \pm 0.08 \dagger$ \\
\hline
\end{tabular}

* Significance of differences between $\mathrm{pH}$ within a feeding group by paired $t$ test, $p<0.01$.

† Significance of differences between feeding groups at the same pH by $t$ test, $p<0.01$. 
Table 3. VFA production ( $\mu$ mol/g wet wt/h), VFA molar proportions ( $\mathrm{mol} / \mathrm{mol}$ lactose fermented), and calculated contributions of different fermentative pathways during incubations of feces (with added lactose, at pH 6.8 and 5.5) of formula-fed (FF) and breast-fed (BF) infants (mean \pm SEM)

\begin{tabular}{|c|c|c|c|}
\hline & \multicolumn{3}{|c|}{ VFA production } \\
\hline & Lactate & Acetate & Propionate \\
\hline \multicolumn{4}{|c|}{$\mathrm{pH} 6.8$} \\
\hline $\mathrm{FF}$ & $102 \pm 21$ & $143 \pm 31$ & $18 \pm 3$ \\
\hline $\mathrm{BF}$ & $106 \pm 8$ & $112 \pm 25$ & $4 \pm 2 *$ \\
\hline \multicolumn{4}{|c|}{ pH 5.5} \\
\hline $\mathrm{FF}$ & $78 \pm 18$ & $88 \pm 23$ & $14 \pm 3$ \\
\hline $\mathrm{BF}$ & $79 \pm 17$ & $75 \pm 21$ & $2 \pm 1$ \\
\hline \multicolumn{4}{|c|}{$\begin{array}{l}\text { VFA molar proportions } \\
\text { pH } 6.8\end{array}$} \\
\hline FF & $1.9 \pm 0.1$ & $2.6 \pm 0.2$ & $0.37 \pm 0.05$ \\
\hline $\mathrm{BF}$ & $2.3 \pm 0.2$ & $2.4 \pm 0.2$ & $0.09 \pm 0.05^{*}$ \\
\hline \multicolumn{4}{|c|}{ pH 5.5} \\
\hline $\mathrm{FF}$ & $2.1 \pm 0.2$ & $2.3 \pm 0.2$ & $0.21 \pm 0.13$ \\
\hline \multirow[t]{3}{*}{$\mathrm{BF}$} & $2.4 \pm 0.5$ & $2.3 \pm 0.3$ & $0.06 \pm 0.01$ \\
\hline & \multicolumn{3}{|c|}{ Contribution of pathway to total VFA } \\
\hline & Bifidobacterial & Homolactate & Propionate \\
\hline \multicolumn{4}{|c|}{$\mathrm{pH} 6.8$} \\
\hline $\mathrm{FF}$ & $0.62 \pm 0.09$ & $0.14 \pm 0.05$ & $0.25 \pm 0.08$ \\
\hline $\mathrm{BF}$ & $0.64 \pm 0.10$ & $0.29 \pm 0.08^{*}$ & $0.09 \pm 0.04^{*}$ \\
\hline \multicolumn{4}{|c|}{ pH 5.5} \\
\hline $\mathrm{FF}$ & $0.53 \pm 0.10 \dagger$ & $0.30 \pm 0.05 \dagger$ & $0.17 \pm 0.05$ \\
\hline $\mathrm{BF}$ & $0.51 \pm 0.08 \uparrow$ & $0.42 \pm 0.07 \dagger$ & $0.07 \pm 0.06$ \\
\hline
\end{tabular}

* Significance of differences between feeding groups within a $\mathrm{pH}, p<$ 0.05 by unpaired $t$ test.

$\dagger$ Significance of differences within a feeding group, but with different $\mathrm{pH}, p<0.05$ by paired $t$ test.

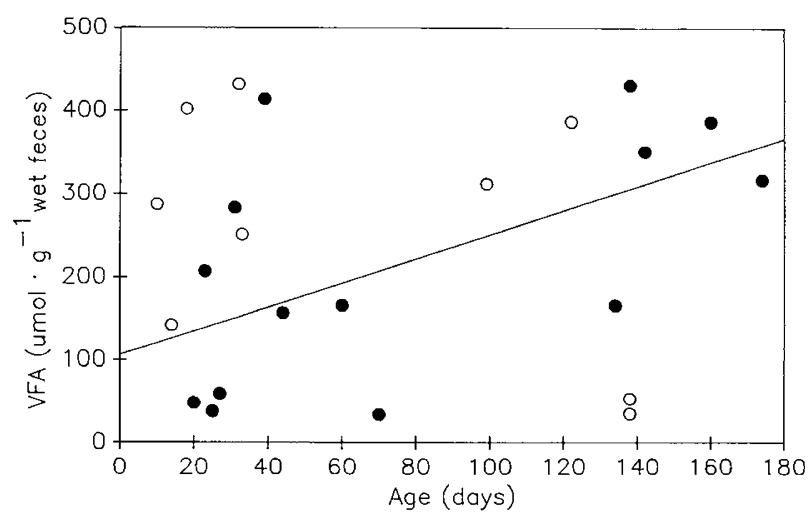

Fig. 1. Relationship between the age of the infants and the sum of the VFA present in stools at the end of 1-h incubation at $\mathrm{pH} 6.8$, after the addition of lactose. Results of 1-h incubation added lactose were subtracted. A significant positive correlation was found for breast-fed infants $(\bullet), p=0.03$, but not for formula fed infants $(\bigcirc)$. The regression line is derived from the data from breast-fed infants.

breast-fed infants process the carbohydrate that arrives in the large bowel during a diarrheal episode (16). Unfermented carbohydrate in the colonic lumen produces an osmolar load that leads to water secretion and diarrhea.

In our study, additional lactose was readily hydrolyzed and fermented by bacteria in the feces of both groups of infants. Acidification and incubation of the feces, however, produced some differences between groups. In acidifying the feces, we chose a pH of 5.5 because it inhibited $\mathrm{H}_{2}$ production in fecal incubates to which $\mathrm{CHO}$ had been added, as demonstrated by Perman et al. (17). In vivo, when the amount of CHO that arrives in the colon is larger than normal, bacteria ferment it to lactic acid and VFA. Under such conditions, a fecal pH of 5.5 or less can be observed in both breast-fed and formula-fed infants. To determine whether the accumulation of product (acetate) also had an effect on hydrolysis and fermentation, we used two different substances to acidify each stool homegenate: acetate and $\mathrm{HCl}$. We observed no significant differences in results obtained from acetate-acidified incubates compared with those obtained from $\mathrm{HCl}$-acidified incubates, which indicated that $\mathrm{pH}$ was the important variable with regard to changes in hydrolysis and fermentation.

We observed that the feces of breast-fed infants hydrolyzed significantly less lactose at $\mathrm{pH} 5.5(84 \mu \mathrm{mol} / \mathrm{g})$ than at $\mathrm{pH} 6.8$ $(99 \mu \mathrm{mol} / \mathrm{g})$, whereas the amount of lactose hydrolyzed by the feces of formula-fed infants was not significantly affected by the change in $\mathrm{pH}$. In addition, at $\mathrm{pH} 5.5$ the proportion of the hydrolyzed lactose that was fermented decreased by $25 \%$ and the concentration of hexose increased in both feeding groups, in parallel with the decline in the amount of VFA produced. The increase in hexose concentration was particularly marked in the feces from formula-fed infants, because the decrease in fermentation was not balanced by a significant decrease of lactose hydrolysis.

These observed differences could be explained by differences in the $\mathrm{pK}$ of bacterial enzymes. A rapid inhibition of hydrolytic enzymes in the fecal bacteria of breast-fed infants may be responsible for the observed lactose accumulation. Our results indicate that an acid $\mathrm{pH}$ in the feces of breast-fed infants primarily inhibits disaccharide hydrolysis by the flora, whereas the same $\mathrm{pH}$ in the feces of formula-fed infants primarily inhibits fermentation by the flora. If this observation were true in vivo, i.e. if lactose, rather than its constituent monosaccharides, accumulated in the colonic lumen of breast-fed infants with diarrhea, these infants would have the advantage of a lower osmolar load compared with that of formula-fed infants.

The mean total calculated osmolarity in our samples was 300 mosmol/L for the incubates of formula-fed infants and 244 mosmol/L for breast-fed infants. The in vitro incubation system, however, does not allow for the disappearance of formed VFA, which occurs in the colon. In addition, the amount of lactose that we added to the incubates was arbitrary, and differences in total colonic content (particularly qualitative and quantitative

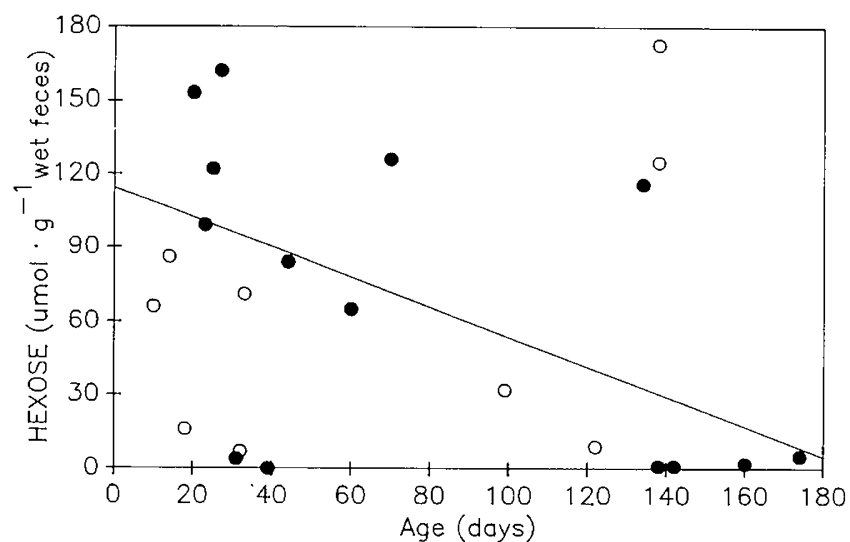

Fig. 2. Relationship between the age of the infants and the hexose remaining in stools at the end of 1-h incubation at $\mathrm{pH} 6.8$, after the addition of lactose. Results of 1 -h incubation without added lactose were subtracted. A significant negative correlation was found for breast-fed infants $(\bullet), p=0.03$, but not for formula-fed infants $(O)$. The regression line is derived from the data from breast-fed infants. 


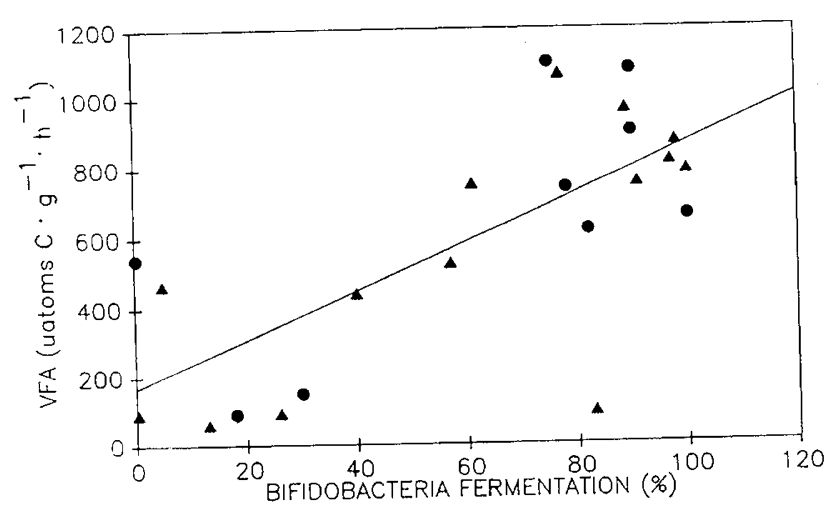

Fig. 3. Relationship between the fermentation pattern and overall rate of fermentation. Variations in total fermentation (VFA present in the incubate at $\mathrm{pH} 6.8$, after the addition of lactose; results from the incubation without added lactose were subtracted) were closely related to the proportion of the fermentation accounted for by bifidobacteria for both formula-fed $(\bullet)$ and breast-fed infants $(\boldsymbol{\Lambda})$. The regression line is the mean for the data from both the breast-fed and formula-fed infants.

differences in the bacteria) of breast-fed and formula-fed infants, however, could affect carbohydrate fermentation. We do not know, therefore, whether our hypothesis would hold true in vivo. Studies in vivo using stable isotopes may help us resolve this question.

We observed a considerable range in the rates of fermentation, especially in the feces from breast-fed infants. At $\mathrm{pH}$ 6.8, fecal incubates from formula-fed infants hydrolyzed a mean 102 $\mu \mathrm{mol} / \mathrm{g}$ and fermented a mean $55 \mu \mathrm{mol} / \mathrm{g}$, amounts that were $\mu \mathrm{mol} / \mathrm{g}$ and ferme from incubates of breast-fed infants (99 and 49 $\mu \mathrm{mol} / \mathrm{g}$, respectively). When we examined the relationship be$\mu \mathrm{mol} / \mathrm{g}$, respectiven fermentation pattern and overall rate of fermentation (Fig. 3 ), it appeared that variations in total fermentation were closely related to the proportion of the fermentation accounted for by bifidobacteria.

We chose not to present the data for formic acid, because it did not contribute to an understanding of the fermentation process. Formic acid disappeared in the incubations of the formula-fed group, both in the presence and absence of additional lactose. Formic acid was not significantly changed in the incubations of feces from breast-fed infants. Formic acid can be converted to $\mathrm{H}_{2}$ and $\mathrm{CO}_{2}$ (which we did not measure) and can also be converted to acetic acid (one acetic acid per four formic acids). Although we recognize that VFA interconversions may have occurred, our experimental design prevented us from investigating that possibility.

Our study confirms the findings of Gerstley and coworkers $(10,11)$ that the content of VFA and propionic acid is higher in the feces of formula-fed infants compared with that of breast-fed infants. Gerstley et al. (11) did not find a correlation between VFA content and the age of the child. We found a positive correlation for the breast-fed infants, but no association for the formula-fed infants (Fig. 1). We also observed a negative correlation between age and the amount of monosaccharides after a 1-h incubation with added lactose (Fig. 2). The fecal flora of the formula-fed infant seems to establish itself earlier and to resemble more that of adults than does the flora of exclusively breast-fed infants. Stark and Lee (12), who examined the bacterial population in the large bowel of seven formula-fed and seven breastfed infants, found that the introduction of solid foods to breastfed infants caused a major change in the microbial ecology of the large bowel: counts of enterobacteria and enterococci rose sharply, and colonization by bacteroides, clostridia, and anaerobic streptococci occurred (12). This change in microbial ecology was not observed when solid foods were introduced into the diets of formula-fed infants; counts of facultative anaerobes remained high, whereas colonization by anaerobes other than bifidobacteria continued.

These findings support the hypothesis that the growth and development of the fecal flora occur early in the life of formulafed infants and is independent of the introduction of dietary solids. More recent studies, however, have failed to demonstrate a bifidobacteria-predominant flora in the feces of breast-fed infants who live in developed countries $(4,5)$. Instead, coliforms and bacteroides were the predominant organisms. In a recent study by Midtvedt et al. (7), the development of several metabolic activities associated with the intestinal microflora were described. Of all the activities studied (production of VFA, degradation of mucin, conversion of bilirubin to urobiolinogen and of cholesterol to coprostanol, inactivation of fecal trypsin, and fermentation of $\mathrm{CHO}$ ) the fermentation of $\mathrm{CHO}$ was the function that developed earliest in life. Midtvedt et al. (7) also found, as we did, that acetate was the predominant VFA in the feces of young infants.

In summary, our results suggest that both the overall rate and the pattern of fermentation are different in the feces of the formula-fed infants compared with those in the feces of the breast-fed group. The formula-fed infants were characterized by a higher production of propionate, the breast-fed infants by a higher level of homolactate fermentation. Under acidic conditions, the hydrolysis of lactose is significantly inhibited in the feces of breast-fed infants and only mildly inhibited in the feces of formula-fed infants. Our results imply a difference in the way in which formula-fed and breast-fed infants respond to the arrival of large loads of $\mathrm{CHO}$ in the colon. This differing response may help explain the observation that diarrheal episodes in acute gastroenteritis of breast-fed infants tend to be less severe than those of formula-fed infants.

\section{REFERENCES}

1. Mata JJ Urrutia JJ 1971 Intestinal colonization of breast fed children in a rural area of low socioeconomic level. Ann NY Acad Sci 176:93-109

2. Willis AT, Bullen CL, Williams K, Fagg CG, Bourne A, Vignon M 1973 Breast milk substitute: a bacteriological study. Br Med J 4:67-72

3. Bullon infants: an in vivo study. J Med Microbiol 9:325-333

4. Mitsuoka T, Kaneuchi C 1977 Ecology of the bifidobacteria. Am J Clin Nutr 30:1799-1810

5. Simhon A, Douglas JR, Drasar BS, Soothill JF 1982 Effect of feeding on infants' faecal flora. Arch Dis Child 57:54-58

6. Lundequist B, Nord CE, Winberg J 1985 The composition of the faecal microflora in breastfed and bottle fed infants from birth to eight weeks. Acta Paediatr Scand 74:45-51

7. Midtvedt T, Carlstedt-Duke B, Hoverstad T, Lingaas E, Norin E, Saxerholt H, Steinbakk M 1986 Influence of peroral antibiotics upon the biotransformatory activity of the intestinal microflora in healthy subjects. Eur J Clin Invest 16:11-17

8. Chen HM, Lifschitz CH 1989 Preparation of fecal samples for assay of volatile fatty acids by gas-liquid chromatography and high-performance liquid chromatography. Clin Chem 35:74-76

9. Wolin MJ 1981 Fermentation in the rumen and human large intestine. Science 213:1463-1468

10. Gerstley JR, Wang CC, Boyden RE, Wood AA 1928 The influence of feeding on certain acids in the feces of infants. Am J Dis Child 35:580-589

11. Gerstley JR, Wang CC, Wood AA 1930 Influence of feeding on certain acids in feces of infants. Am J Dis Child 39:487-492

12. Stark PL, Lee A 1982 The microbial ecology of the large bowel of breast-fed and formula-fed infants during the first year of life. J Med Microbiol 15:189199

13. Ross CAC, Dawes EA 1954 Resistance of the breast-fed infant to gastroenteritis. Lancet 1:994-998

14. Bullen CL, Willis AT 1971 Resistance of the breast-fed infant to gastroenteritis Br Med J 3:338-343

15. Duffy LC, Riepenhoff-Talty M, Bears TE, La Scolea LJ, Zielezny MA, Dryja DM, Ogra PL 1986 Modulation of rotavirus enteritis during breast-feeding. Am J Dis Child 140:1164-1168

16. Welsh JK, May JT 1979 Anti-infective properties of breast milk. J Pediatr $94: 1-9$

17. Perman JA, Modler S, Olson AC 1981 Role of $\mathrm{pH}$ in production of hydrogen from Invest 67:643-650 